\title{
A fast, parallel performance of fourth order iterative algorithm on shared memory multiprocessors (SMP) architecture
}

\begin{abstract}
The rotated fourth order iterative algorithm of $\mathrm{O}(\mathrm{h} 4)$ accuracy which was applied to the linear system was introduced by Othman et al. [OTH01] and it was shown to be the fastest compared to the standard fourth order iterative algorithm. Meanwhile the parallel standard fourth order iterative algorithms with difference strategies were implemented successfully by many researchers for solving large scientific and engineering problems. In this paper, the implementation of the parallel rotated fourth order iterative algorithm on SMP architecture is discussed. The performance results of all the parallel algorithms were compared in order to show their outstanding performances.
\end{abstract}

Keyword: Fourth order iterative algorithm; Shared memory multiprocessors 